\title{
Least Square Plane and Leastsquare Quadric Surface Approximation by Using Modified Lagrange's Method
}

\author{
C.V.Rao, \\ GS Department Assistant professor, JubailIndustrial College, Jubail, KSA.
}

\begin{abstract}
Now a days Surface fitting is applied all engineering and medical fields. Kamron Saniee ,2007 find a simple expression for multivariate LaGrange's Interpolation. We derive a least square plane and least square quadric surface Approximation from a given $N+1$ tabular points when the function is unique. We used least square method technique. We can apply this method in surface fitting also.
\end{abstract}

Keywords: Least square, quadric surface, Normal equations

\section{Introduction}

Least square principle method is one the best approximation methodin numerical analysis for line and curve fitting, this method was invented by Lagrange's. A function $y=f(x)$ may be given in discretedata $\left(x_{k}, y_{k}\right)$.The best approximation in the least square is defined as that for which the constants $c_{i}, i=$ $0,1,2,3 \ldots$ nare determined so that the aggregate of $w(x) E$ 2over given domain $\mathrm{D}$ is as small as possible, where $w(x)>0 \$$ is the weight function for the function whose values are given at $N+1$ points $x_{0}, x_{1} \ldots x_{N}$.

We have

$I\left(c_{0}, c_{1} \ldots c_{N}\right)=\sum_{k=o}^{N} w\left(x_{k}\right)\left[f\left(x_{k}\right)-\sum_{i=0}^{n} \emptyset_{i}\left(x_{k}\right)\right]^{2}=$ minimum

Where $\emptyset_{i}(x)=x^{i}, i=0,1,2,3 \ldots n \quad$ and $w(x)=1$

The necessary conditions for (1) to have a minimum value is that

$\frac{\partial I}{\partial c_{i}}=0, i=0,1,2,3 \ldots n$.

This gives a system of $n+1$ linear equations in $n+1$ constants. These equations are called normal equations.

Then we get approximated nth degree polynomial function of $x$.

1.Least square plane: Given a discrete data $\left(x_{k}, y_{k}\right), k=0,1, \ldots N, \quad N \geq 2$.

Consider $\emptyset(x, y)=c_{0}+c_{1} x+c_{2} y$

Such that $I\left(c_{0}, c_{1}, c_{2}\right)=\sum_{k=o}^{N}\left[z_{k}-\left(c_{0}+c_{1} x_{k}+c_{2} y_{k}\right)\right]^{2}=$ minimum

Then Normal equations $\quad \frac{\partial I}{\partial c_{i}}=0, i=0,1,2$.

ie.,

$$
\begin{aligned}
c_{0}(N+1)+c_{1} \sum x_{k}+c_{2} \sum y_{k} & =\sum z_{k} \\
c_{0} \sum x_{k}+c_{1} \sum x_{k}{ }^{2}+c_{2} \sum x_{k} y_{k} & =\sum x_{k} z_{k}
\end{aligned}
$$

$c_{0} \sum y_{k}+c_{1} \sum x_{k} y_{k}+c_{2} \sum y_{k}^{2}=\sum y_{k} z_{k}$

Solving this system of equations we get $c_{0}, c_{1}$ and $c_{2}$.

Example 1: Suppose that given data points $(-1,1,-2),(1,2,3),(1,1,2)$

that lie on $z=f(x, y)$.These points define uniquely a linear function in two variables,

$\operatorname{soz}_{k}=c_{0}+c_{1} x_{k}+c_{2} y_{k} \quad, k=0,1,2$ 
The coefficients satisfy the normal equations

$$
\begin{aligned}
& 3 c_{0}+1 c_{1}+4 c_{2}=3 \\
& c_{0}+3 c_{1}+2 c_{2}=7 \\
& 4 c_{0}+2 c_{1}+6 c_{2}=6
\end{aligned}
$$

Solving these equations we get $c_{0}=-1, c_{1}=2, c_{2}=1$

Thus $z=-1+2 x+y$.

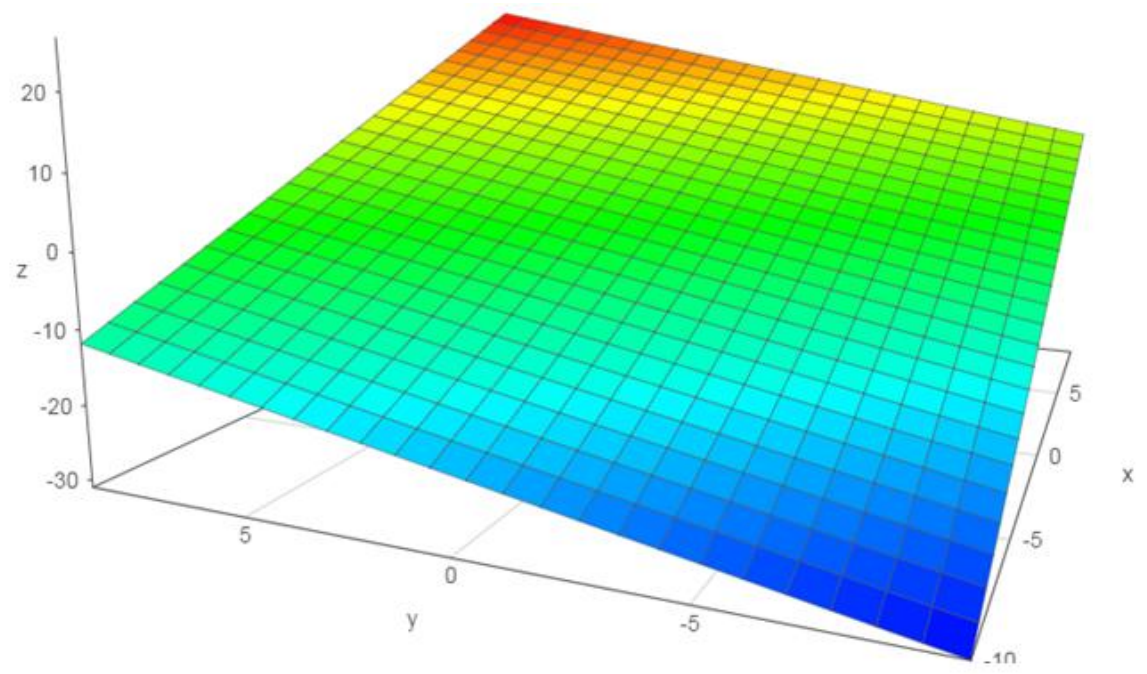

\section{Least square quadric surface}

Given a discrete data $\left(x_{k}, y_{k}\right), k=0,1, \ldots N, \quad N \geq 5$

Consider $\emptyset(x, y)=c_{0}+c_{1} x+c_{2} y+c_{3} x^{2}+c_{4} y^{2}+c_{5} x y$

Such that

$$
I\left(c_{0}, c_{1}, c_{2}, c_{3}, c_{4}, c_{5}\right)=\sum_{k=0}^{N}\left[z_{k}-\left(c_{0}+c_{1} x_{k}+c_{2} y_{k}+c_{3} x_{k}^{2}+c_{4} y_{k}^{2}+c_{5} x_{k} y_{k}\right)\right]^{2}=\text { minimum }
$$

The coefficients satisfy normal equations $\frac{\partial I}{\partial c_{i}}=0, i=0,1,2,3,4,5$.

$$
c_{0}(N+1)+c_{1} \sum x_{k}+c_{2} \sum y_{k}+c_{3} \sum x_{k}^{2}+c_{4} \sum y_{k}^{2}+c_{3} \sum x_{k} y_{k}=\sum z_{k}
$$

$c_{0} \sum x_{k}+c_{1} \sum x_{k}^{2}+c_{2} \sum x_{k} y_{k}+c_{3} \sum x_{k}^{3}+c_{4} \sum x_{k} y_{k}^{2}+c_{5} \sum x_{k}^{2}=\sum x_{k} z_{k}$

$c_{0} \sum y_{k}+c_{1} \sum x_{k} y_{k}+c_{2} \sum y_{k}^{2}+c_{3} \sum x_{k}^{2} y_{k}+c_{4} \sum y_{k}^{3}+c_{5} \sum x_{k} y_{k}^{2}=\sum y_{k} z_{k}$

$c_{0} \sum x_{k}^{2}+c_{1} \sum x_{k}^{3}+c_{2} \sum x_{k}^{2} y_{k}+c_{3} \sum x_{k}^{4}+c_{4} \sum x_{k}^{2} y_{k}^{2}+c_{5} \sum x_{k}^{3} y_{k}=\sum x_{k}^{2} z_{k}$

$c_{0} \sum{y_{k}}^{2}+c_{1} \sum x_{k} y_{k}^{2}+c_{2} \sum y_{k}^{3}+c_{3} \sum x_{k}^{2}{y_{k}}^{2}+c_{4} \sum y_{k}^{4}+c_{5} \sum x_{k} y_{k}^{3}=\sum y_{k}^{2} z_{k}$

$c_{0} \sum x_{k} y_{k}+c_{1} \sum x_{k}{ }^{2} y_{k}+c_{2} \sum x_{k} y_{k}{ }^{2}+c_{3} \sum x_{k}{ }^{3} y_{k}+c_{4} \sum x_{k} y_{k}{ }^{3}+c_{5} \sum x_{k}{ }^{2} y_{k}{ }^{2}=\sum x_{k} y_{k} z_{k}$

Solve these equations we get $c_{0}, c_{1}, c_{2}, c_{3}, c_{4}, c_{5}$.

Example 2: Suppose that given data points $(0,0,0),(0,1,-4),(1,-1,1)$ 
$(1,2,-2),(2,1,4),(1,3,-3)$ those lie on $z=f(x, y)$.These data points satisfy uniquely a degree of two variable function.

$z_{k}=c_{0}+c_{1} x_{k}+c_{2} x_{k}+c_{3} x_{k}^{2}+c_{4} y_{k}^{2}+c_{5} x_{k} y_{k}, k=0,1,2,3,4,5$

The coefficients $c_{0}, c_{1}, c_{2}, c_{3}, c_{4}, c_{5}$ satisfy the normal equations

$$
\begin{gathered}
6 c_{0}+5 c_{1}+6 c_{2}+7 c_{3}+16 c_{4}+6 c_{5}=-4 \\
5 c_{0}+7 c_{1}+6 c_{2}+11 c_{3}+16 c_{4}+8 c_{5}=4 \\
6 c_{0}+6 c_{1}+16 c_{2}+8 c_{3}+36 c_{4}+16 c_{5}=-14 \\
7 c_{0}+11 c_{1}+8 c_{2}+19 c_{3}+18 c_{4}+12 c_{5}=12 \\
16 c_{0}+16 c_{1}+36 c_{2}+18 c_{3}+100 c_{4}+36 c_{5}=-34 \\
6 c_{0}+8 c_{1}+16 c_{2}+12 c_{3}+36 c_{4}+18 c_{5}=-6
\end{gathered}
$$

Solving this system of equations we get

$c_{0}=0, c_{1}=-1, c_{2}=-4, c_{3}=1, c_{4}=0, c_{5}=3$

Thus $z=-x-4 y+x^{2}+3 x y$

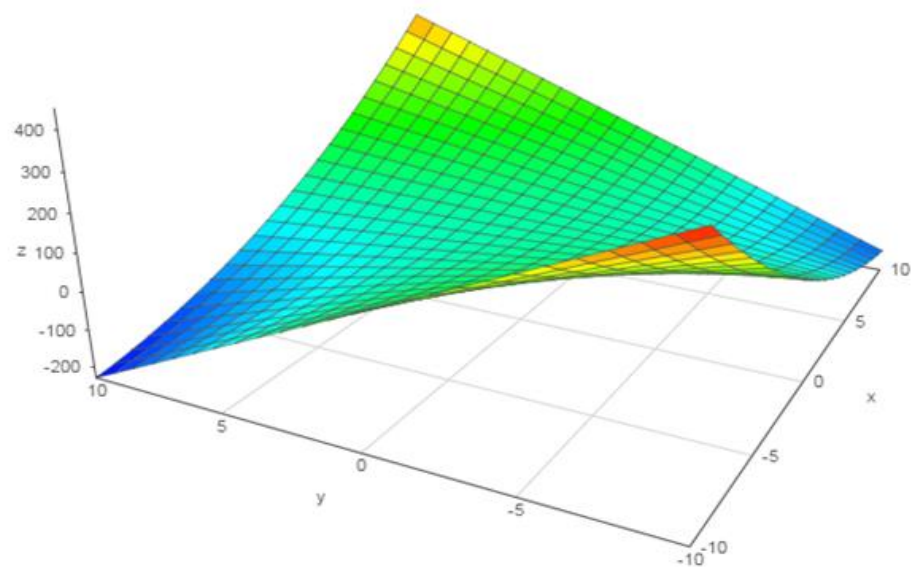

References

[1]. M.K.JAIN ,S.R.KIYENGAR AND R.K.JAIN .Numerical methods for scientific and Engineering Computation ,Wiley Eastern Limited , 1985.

[2]. M. Gasca and T. Sauer.Polynomial interpolation in several variables. Advances in Computationalv Mathematics, 12(4):377-410, 2000

[3]. P.J. Olver. On multivariate interpolation. Studies in Applied Mathematics, 116(4):201-240, 2006.

[4]. J.F. Steffensen. Interpolation.Dover Publications, Inc., New York, second edition, 2006. Copyright ()

[5]. Kamron Saniee, A simple expression for multivariate Lagrange Interpolation , 2007. 